\title{
Reduction of the number of material parameters by ANN approximation
}

\author{
Wojciech Sumelka • Tomasz Lodygowski
}

Received: 6 November 2011 / Accepted: 18 October 2012 / Published online: 10 November 2012

(C) The Author(s) 2012. This article is published with open access at Springerlink.com

\begin{abstract}
Modern industrial standards require advanced constitutive modeling to obtain satisfactory numerical results. This approach however, is causing significant increase in number of material parameters which can not be easily obtained from standard and commonly known experimental techniques. Therefore, it is desirable to introduce procedure decreasing the number of the material parameters. This reduction however, should not lead to misunderstanding the fundamental physical phenomena. This paper proposes the reduction of the number of material parameters by using ANN approximation. Recently proposed viscoplasticity formulation for anisotropic solids (metals) developed by authors is used as an illustrative example. In this model one needs to identify 28 material parameters to handle particular metal behaviour under adiabatic conditions as reported by Glema et al (J Theor Appl Mech 48:973-1001, 2010), (Int J Damage Mech 18:205-231, 2009) and Sumelka (Poznan University of Technology, Poznan, 2009). As a result of proposed approach, authors decreased the number of material parameters to 19 .
\end{abstract}

Keywords Material parameters · Anisotropy · Viscoplasticity $\cdot$ ANN approximation

\footnotetext{
W. Sumelka $(\varangle) \cdot T$. Łodygowski

Division of Computer Aided Design, Faculty of Civil and Environmental Engineering, Institute of Structural Engineering, Poznan University of Technology, ul. Piotrowo 5, 60-965 Poznań Poland

e-mail: wojciech.sumelka@put.poznan.pl

T. Łodygowski

e-mail: Tomasz.Lodygowski@ put.poznan.pl
}

\section{Introduction}

The complexity of mathematical aspect of metal behavior modeling maintain on rising in order to match recent industrial requirements (especially thermo-mechanical coupling including damage). The modeling must reflect micro/nano scale of observation in order to capture physical phenomena crucial for its proper description $[6,37]$. As a result, the modern constitutive models include high number of material parameters. For example, well established and relatively simple constitutive model proposed by Johnson and Cook [10] requires identification of 14 material parameters to describe metal behavior. More sophisticated models, such as Rusinek-Klepaczko model [30-32] or recently proposed model to analyze hypervelocity projectile-target impact [4] require identification up to 19 (depending on analyzed process) and even 30 material parameters, respectively.

The experimental (or mathematical) identification of such models, if at all possible, can be very expensive (in its common or computational time sense). Hence it is obvious that the reduction of the number of material parameters, without loosing particular model generality, is advisable. In order to meet such demands, authors present in this paper the general procedure of using the artificial neural network (ANN) for material functions approximation. As an application example of ANN approximation procedure we use recently proposed viscoplasticity formulation for anisotropic solids, showing finally it effects in the reduction of desired material parameters in the model under consideration (from 28 to 19).

This paper is divided into three major parts.

In Sect. 2 the fundamental results of viscoplastic theory formulation for anisotropic solids are presented to understand necessity of further described ANN approximation. In particular we discuss: description of damage phenomena, definition of the body kinematics, constitutive postulates and 
constitutive relations with a complete definition of material functions for adiabatic process.

Section 3 deals with the concept of ANN approximation for selected material functions in the discussed model. The most important result of this section is that we can apply ANN approximation without loosing the physical interpretation of all variables in the model comparing to the classical viscoplasticity formulation by Perzyna (cf. [28]).

Finally, in Sect. 4 the material model implementation in a finite element software Abaqus/Explicit by utilizing VUMAT user subroutine is briefly discussed and its application concerning high speed machining (orthogonal cutting of DH-36 steel sample) is presented.

\section{Main results of viscoplasticity formulation for anisotropic solids}

\subsection{General discussion}

The material model is formulated in terms of the continuum mechanics, in the framework of the thermodynamical theory of viscoplasticity along with the phenomenological approach $[7,29,36]$. Formally, the constitutive structure belongs to the class of simple materials with fading memory, and due to its final form and the way of incorporating the fundamental variables, belongs to the rate type materials with internal state variables [38].

The key features of the formulation (for detailed and more general formulation please see $[6,35,37]$ ) are: (i) the description is invariant with respect to any diffeomorphism (the obtained model is covariant [13]), (ii) the obtained evolution problem is well-posed, (iii) sensitivity to the rate of deformation, (iv) finite elasto-viscoplastic deformations, (v) plastic non-normality, (vi) dissipation effects (anisotropic description of damage), (vii) thermo-mechanical couplings and (viii) length scale sensitivity.

In the discussed model an important role plays the description of damage. We introduce the second order microdamage tensorial field (as a state variable), denoted by $\xi$ cf. $[7,29,35]$, which reflects the experimentally observed anisotropy of metals in the mathematical (constitutive) model. Such approach enables us to maintain high level of the global damage approximation (GDA) (strain-stress curves fitting from experiment and mathematical model) as well as particularly high level of local damage approximation (LDA) (GDA plus coincidence in: macrodamage initiation time, velocity of macrodamage evolution and the geometry of macrodamage pattern). Let us emphasize that the Euclidean norm of the microdamage field defines the scalar quantity called the volume fraction porosity or simply porosity [29] while its principal values are proportional to the ratio of the damaged area and the assumed characteristic area of the representative

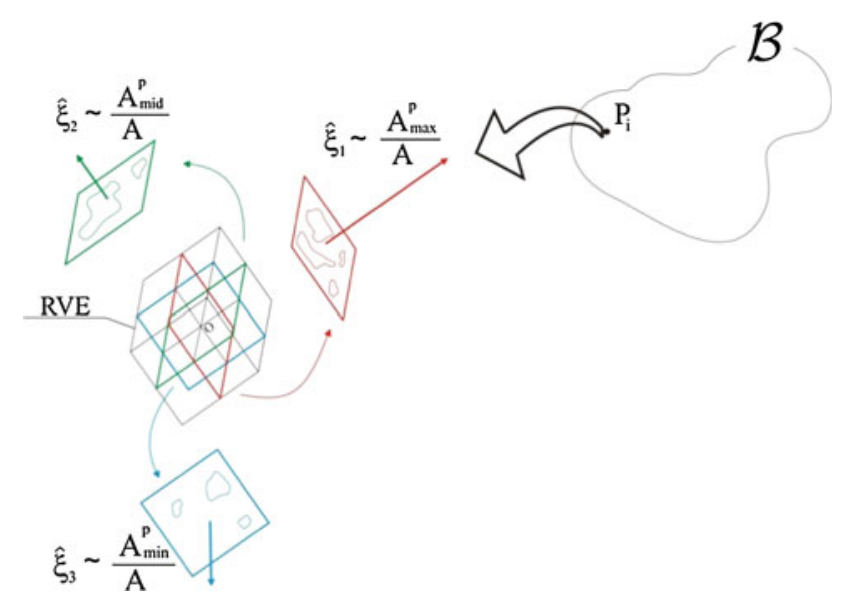

Fig. 1 The concept of microdamage tensor

volume element [35]. Thus, they indicate damage plane as one perpendicular to maximal principal value of $\xi$ (cf. Fig. 1).

\subsection{Adiabatic process}

\section{Kinematics}

The abstract body is a differentiable manifold. The kinematics of the finite elasto-viscoplastic deformations is governed by the multiplicative decomposition of the total deformation gradient to the elastic and viscoplastic parts [12]

$\mathbf{F}(\mathbf{X}, t)=\mathbf{F}^{e}(\mathbf{X}, t) \cdot \mathbf{F}^{p}(\mathbf{X}, t)$,

where (1) $\mathbf{F}=\frac{\partial \phi(\mathbf{X}, t)}{\partial \mathbf{X}}$ is the deformation gradient, $\phi$ describes the motion, $\mathbf{X}$ denotes material coordinates, $t$ represents time and $\mathbf{F}^{e}, \mathbf{F}^{p}$ are elastic and viscoplastic parts of the deformation gradient, respectively.

From the spatial deformation gradient, denoted by $\mathbf{l}$,

$\mathbf{I}(\mathbf{x}, t)=\frac{\partial \boldsymbol{v}(\mathbf{x}, t)}{\partial \mathbf{x}}$,

where $\boldsymbol{v}$ denotes spatial velocity and $\mathbf{x}$ are spatial coordinates, we obtain

$\mathbf{l}=\mathbf{d}+\mathbf{w}=\mathbf{d}^{e}+\mathbf{w}^{e}+\mathbf{d}^{p}+\mathbf{w}^{p}$,

$\mathbf{d}=\frac{1}{2}\left(\mathbf{l}+\mathbf{l}^{T}\right)$,

$\mathbf{w}=\frac{1}{2}\left(\mathbf{l}-\mathbf{l}^{T}\right)$,

where $\mathbf{d}$ is the symmetric part and $\mathbf{w}$ is the antisymmetric part, of $\mathbf{l}$, respectively. Now, assuming that the Euler-Almansi strain is taken as a strain measure and applying Lie derivative we have the fundamental relation

$\mathbf{d}^{\mathrm{b}}=\mathrm{L} \boldsymbol{v}\left(\mathbf{e}^{\mathrm{b}}\right)$,

and simultaneously 

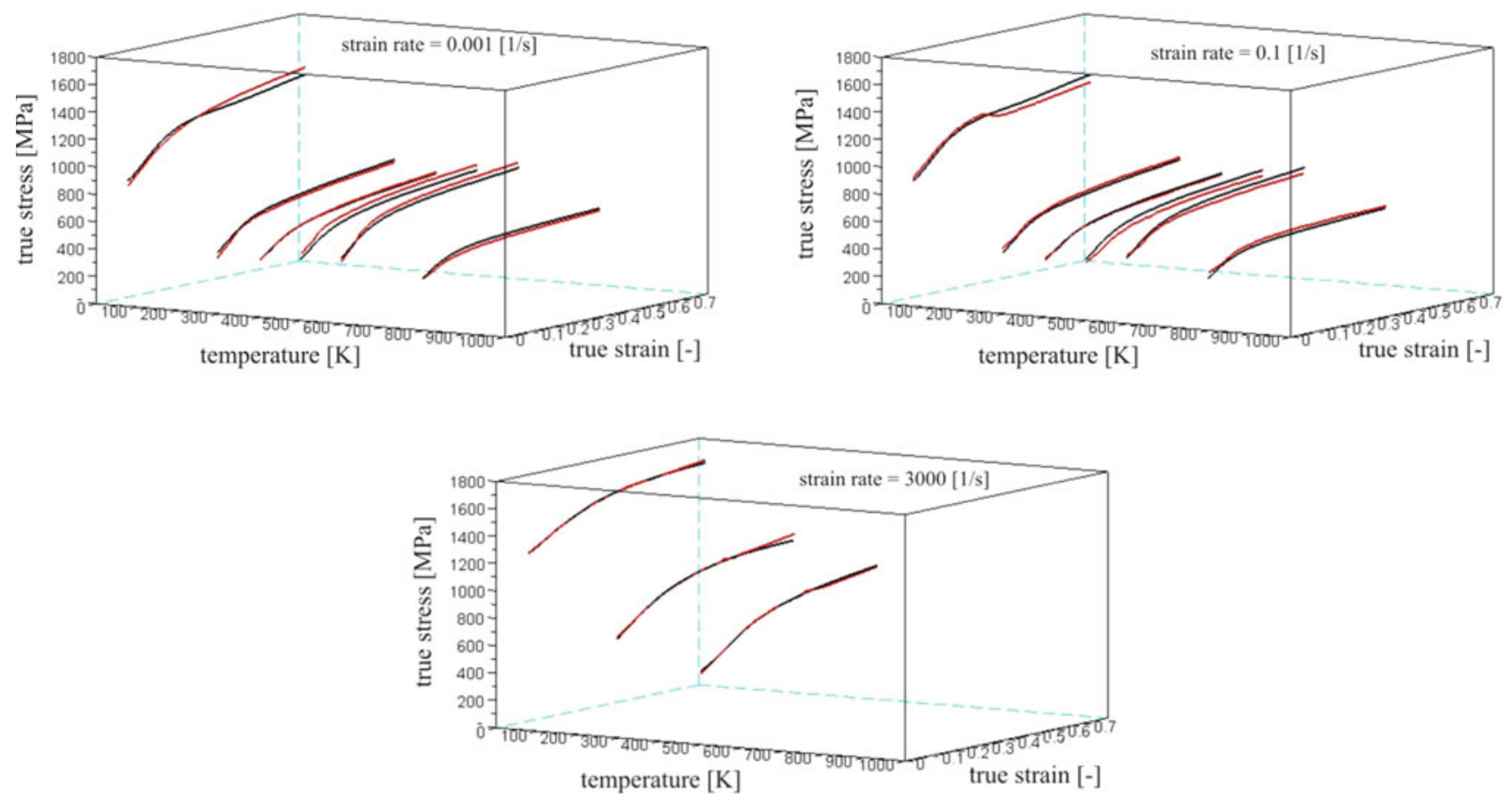

Fig. 2 The strain-stress relations taken from experimental results [15] (red lines) and its ANN approximation (black lines). (Color figure online)
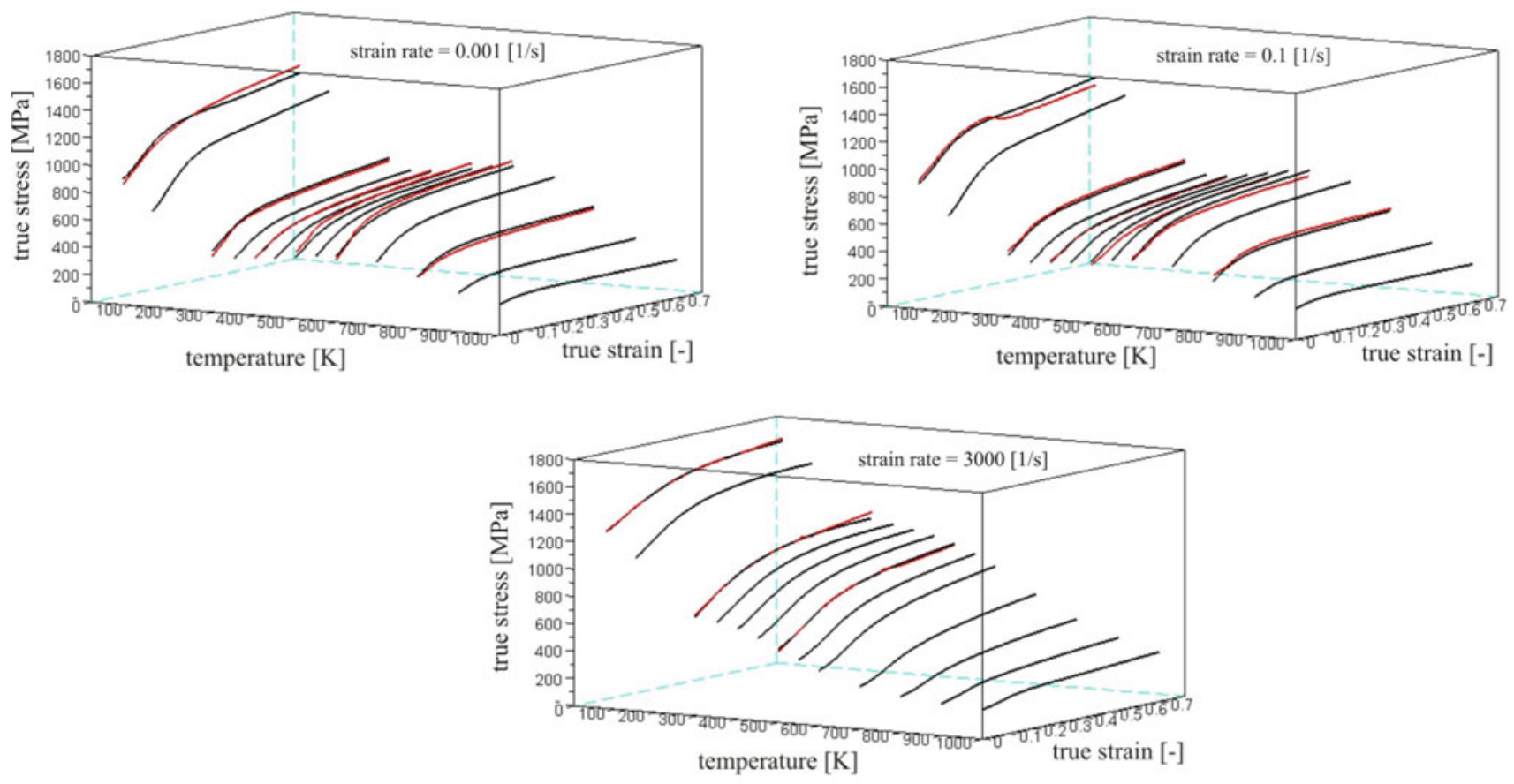

Fig. 3 The strain-stress relations taken from experimental results [15] (red lines) and its ANN approximation/extrapolation (black lines) through the temperature axis. (Color figure online)

$\mathbf{d}^{e b}=\mathrm{L} \boldsymbol{v}\left(\mathbf{e}^{e b}\right), \quad \mathbf{d}^{p b}=\mathrm{L} \boldsymbol{v}\left(\mathbf{e}^{p b}\right)$

where $\mathrm{L}_{\boldsymbol{v}}$ stands for Lie derivative, e stands for the Euler-Almansi strain, b indicates that a tensor has all its indices lowered [13], indices $e$ and $p$ denote elastic and viscoplastic parts, respectively. These relations show that the symmetric part of the spatial deformation gradient $\mathbf{d}$ is directly Lie derivative of the Euler-Almansi strain. 

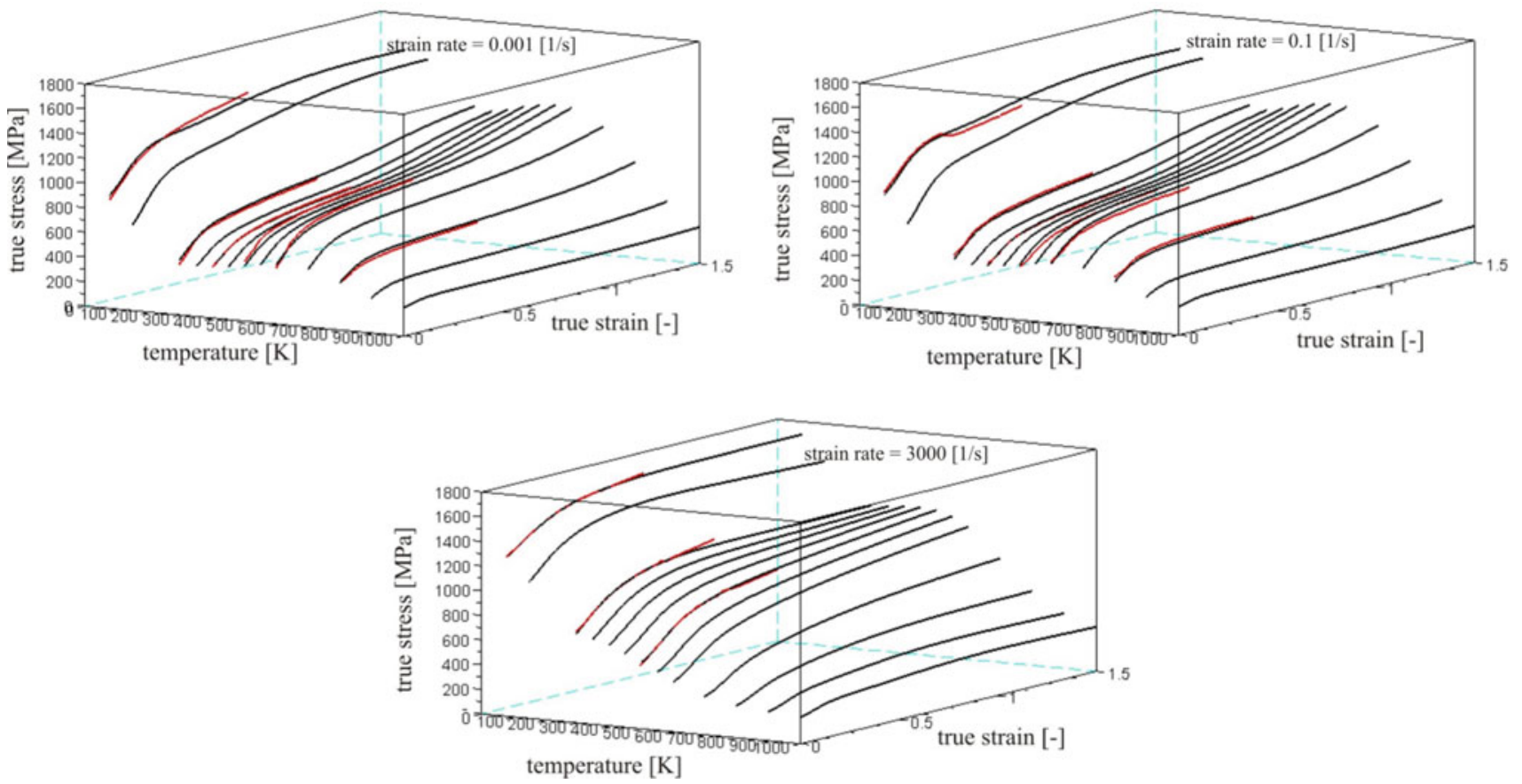

Fig. 4 The strain-stress relations taken from experimental results [15] (red lines) and its ANN approximation/extrapolation (black lines) through the temperature and strains axis. (Color figure online)

\section{Constitutive postulates}

Assuming that the balance principles hold, namely: conservation of mass, balance of momentum, balance of moment of momentum and balance of energy and entropy production, we define four constitutive postulates [26,28]:

(i) Existence of the free energy function $\psi$. Formally we apply it in the following form

$\psi=\hat{\psi}(\mathbf{e}, \mathbf{F}, \vartheta ; \boldsymbol{\mu})$,

where $\boldsymbol{\mu}$ denotes a set of internal state variables governing the description of dissipation effects and $\vartheta$ represents temperature. It is important to notice, that we have used semicolon to separate the last variable due to its different nature (it introduces a dissipation to the model), without $\boldsymbol{\mu}$ the presented model describes thermoelasticity.

(ii) Axiom of objectivity (spatial covariance). The material model should be invariant with respect to any superposed motion (diffeomorphism).

(iii) The axiom of the entropy production. For every regular process the constitutive functions should satisfy the second law of thermodynamics.

(iv) The evolution equation for the internal state variables vector $\boldsymbol{\mu}$ should be of the form
$\mathrm{L} v \boldsymbol{\mu}=\hat{\mathbf{m}}(\mathbf{e}, \mathbf{F}, \vartheta, \boldsymbol{\mu})$

where evolution function $\hat{\mathbf{m}}$ has to be determined based on the experimental observations.

\section{Initial boundary value problem}

Assuming that the above assumptions hold, the deforming body under adiabatic regime is governed by the following set of equations. They state the initial boundary value problem (IBVP).

Find $\phi, \boldsymbol{v}, \rho, \boldsymbol{\tau}, \boldsymbol{\xi}, \vartheta$ as functions of $t$ and position $\mathbf{x}$ such that the following equations are satisfied $[19,21,22,27]$ :

(i) The field equations

$$
\begin{aligned}
& \dot{\phi}=\boldsymbol{v}, \\
& \dot{\boldsymbol{v}}=\frac{1}{\rho_{\operatorname{Ref}}}\left(\operatorname{div} \boldsymbol{\tau}+\frac{\boldsymbol{\tau}}{\rho} \cdot \operatorname{grad} \rho-\frac{\boldsymbol{\tau}}{1-(\boldsymbol{\xi}: \boldsymbol{\xi})^{\frac{1}{2}}} \operatorname{grad}(\boldsymbol{\xi}: \boldsymbol{\xi})^{\frac{1}{2}}\right), \\
& \dot{\rho}=-\rho \operatorname{div} \boldsymbol{v}+\frac{\rho}{1-(\boldsymbol{\xi}: \boldsymbol{\xi})^{\frac{1}{2}}}(\mathrm{~L} \boldsymbol{v} \boldsymbol{\xi}: \mathrm{L} \boldsymbol{v} \boldsymbol{\xi})^{\frac{1}{2}} \\
& \dot{\boldsymbol{\tau}}=\mathcal{L}^{e}: \mathbf{d}+2 \boldsymbol{\tau} \cdot \mathbf{d}-\mathcal{L}^{t h} \dot{\vartheta}-\left(\mathcal{L}^{e}+\mathbf{g} \boldsymbol{\tau}+\boldsymbol{\tau} \mathbf{g}\right): \mathbf{d}^{p}, \\
& \dot{\boldsymbol{\xi}}=2 \xi \cdot \mathbf{d}+\frac{\partial g^{*}}{\partial \boldsymbol{\tau}} \frac{1}{T_{m}}\left\langle\Phi^{g}\left[\frac{I_{g}}{\tau_{e q}(\boldsymbol{\xi}, \vartheta, \in p}-1\right]\right), \\
& \dot{\vartheta}=\frac{\chi^{*}}{\rho c_{p}} \boldsymbol{\tau}: \mathbf{d}^{p}+\frac{\chi^{* *}}{\rho c_{p}} \mathbf{k}: \mathrm{L} \boldsymbol{v} \boldsymbol{\xi},
\end{aligned}
$$



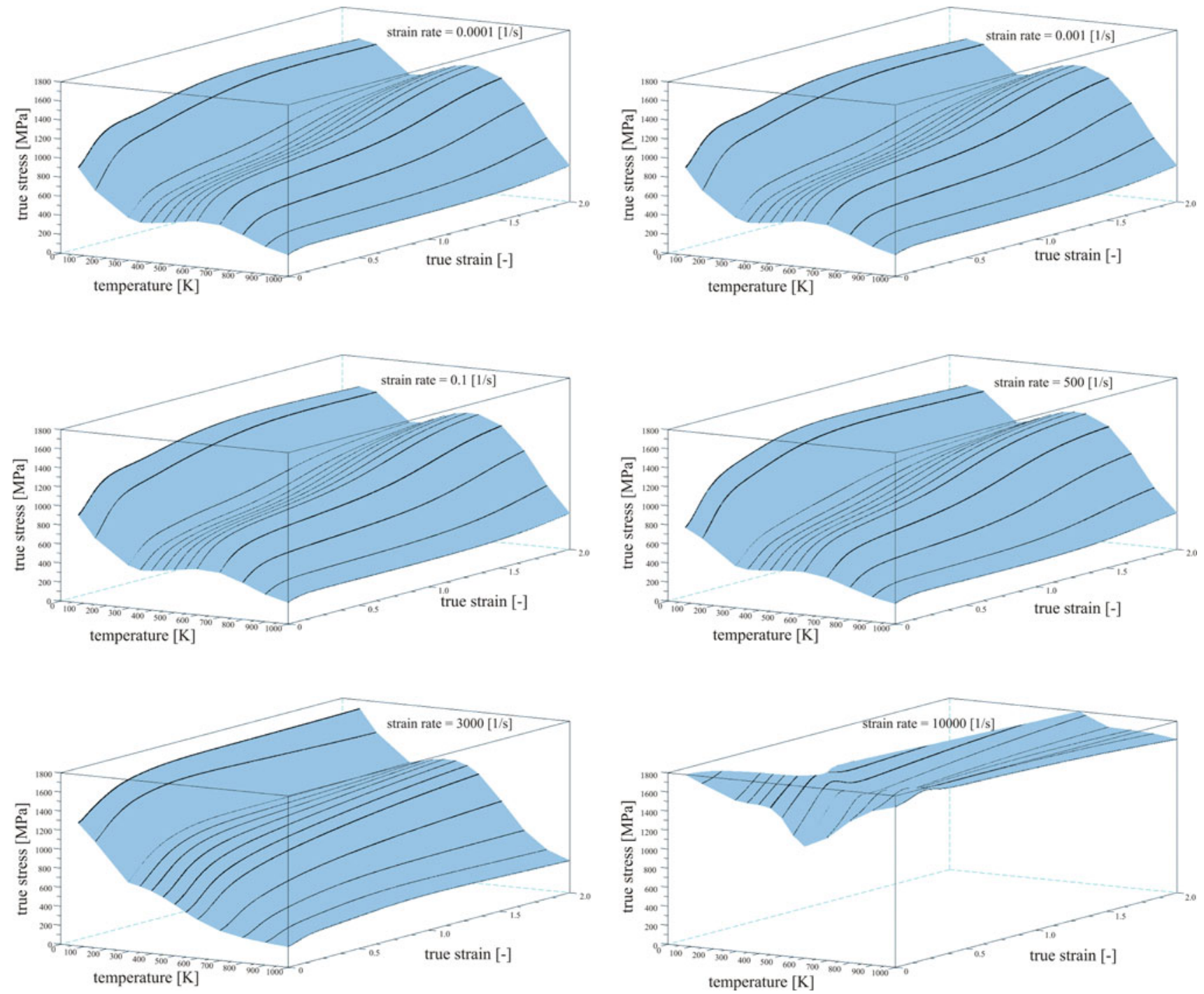

Fig. 5 The flow surfaces through the change of strain rates: experimental results $0.001,0.1,3,000 \mathrm{~s}^{-1}$ [15] and its ANN extrapolation $0.0001,500,10,000 \mathrm{~s}^{-1}$

(ii) The boundary conditions

(a) Displacement $\phi$ is prescribed on a part $\Gamma_{\phi}$ of $\Gamma(\mathcal{B})$ and tractions $(\boldsymbol{\tau} \cdot \mathbf{n})^{a}$ are prescribed on a part $\Gamma_{\boldsymbol{\tau}}$ of $\Gamma(\mathcal{B})$, where $\Gamma_{\phi} \cap \Gamma_{\boldsymbol{\tau}}=0$ and $\Gamma_{\phi} \cup \Gamma_{\boldsymbol{\tau}}=$ $\Gamma(\mathcal{B})$,

(b) Heat flux $\mathbf{q} \cdot \mathbf{n}=0$ is prescribed on $\Gamma(\mathcal{B})$,

(iii) The initial conditions $\phi, \boldsymbol{v}, \rho, \boldsymbol{\tau}, \boldsymbol{\xi}, \vartheta$ are given for each particle $\mathbf{X} \in \mathcal{B}$ at $t=0$,

In above equations we have denoted: $\rho_{\text {Ref }}$ as a referential density, $\boldsymbol{\tau}$ as the Kirchhoff stress tensor, $\rho$ as a current density, $\mathcal{L}^{e}$ as an elastic constitutive tensor, $\mathcal{L}^{\text {th }}$ as a thermal operator, $\mathbf{g}$ as a metric tensor, $\frac{\partial g^{*}}{\partial \boldsymbol{\tau}}$ as the evolution directions for anisotropic microdamage growth processes, $T_{m}$ as a relaxation time of the mechanical disturbances, $I_{g}$ as a stress intensity invariant, $\tau_{e q}$ as the threshold stress, $\chi^{*}, \chi^{* *}$ as the irreversibility coefficients and $c_{p}$ as a specific heat.

\section{Material functions}

For the evolution problem (10) we assume the following:

1. For elastic constitutive tensor $\mathcal{L}^{e}$

$$
\mathcal{L}^{e}=2 \mu \mathcal{I}+\lambda(\mathbf{g} \otimes \mathbf{g})
$$

where $\mu, \lambda$ are Lamé constants.

2. For thermal operator $\mathcal{L}^{\text {th }}$

$$
\mathcal{L}^{\text {th }}=(2 \mu+3 \lambda) \theta \mathbf{g},
$$


Table 1 Material parameters for DH-36 steel - boxed parameters are those being reduced by ANN approximation

\begin{tabular}{llll}
\hline$\lambda=121.154 \mathrm{GPa}$ & $\mu=80.769 \mathrm{GPa}$ & $T_{m}=2.5 \mu \mathrm{s}$ & $m_{p l}=0.13$ \\
$n_{1}=0$ & $n_{2}=0.25$ & $\chi^{*}=1.0$ & $\chi^{* *}=0.8$ \\
$\kappa_{s}^{*}$ & $\kappa_{s}^{* *}$ & $\kappa_{0}^{*}$ & $\kappa_{0}^{* *}$ \\
\hline \hline$\delta^{*}$ & $\delta^{* *}$ & $\beta^{*}=9.0$ & $\beta^{* *}=2.0$ \\
$c=0.067$ & $\theta=10^{-6} \mathrm{~K}^{-1}$ & $m_{g}=1$ & $m_{F}-$ \\
$b_{1}=0.58$ & $b_{2}=0.7$ & $b_{3}=0$ & $\left\|\mathrm{~L} \xi_{c}\right\|-$ \\
$\xi^{F^{*}=0.30}$ & $\xi^{F^{* *}}$ & $c_{p}=470 \mathrm{~J} / \mathrm{kgK}$ & $\rho_{R e f}=7,800 \mathrm{~kg} / \mathrm{m}^{3}$ \\
\hline
\end{tabular}

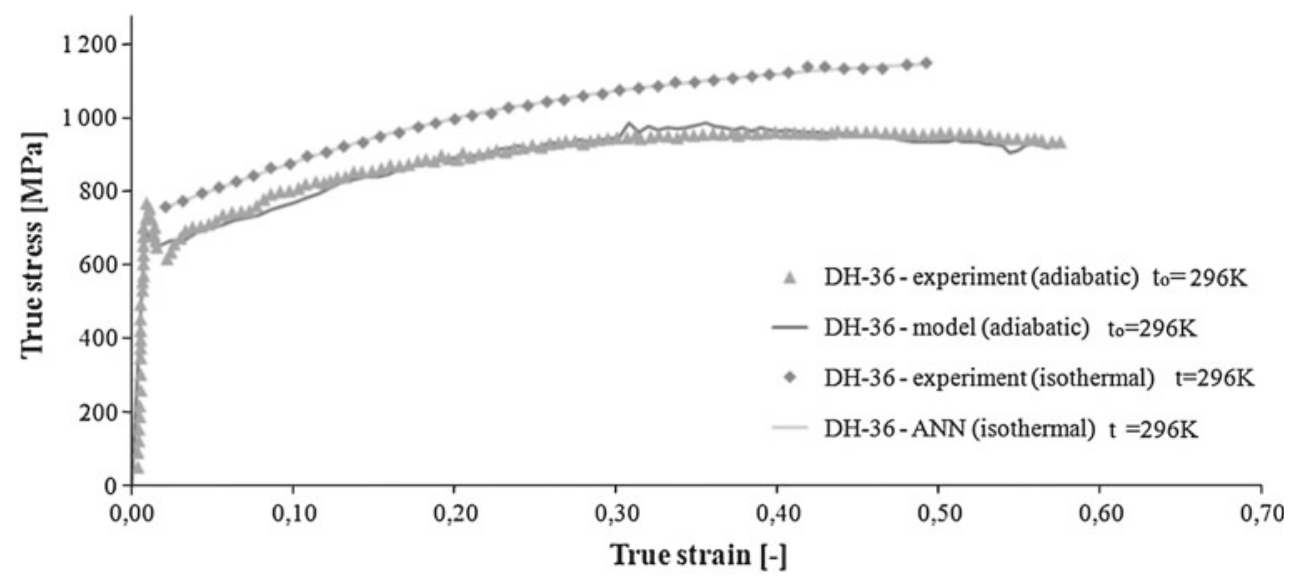

Fig. 6 The comparison of strain-stress curves (isothermal and adiabatic) from the experiment and the model for a strain rate $3,000 \mathrm{~s}^{-1}$ and initial temperature of $296 \mathrm{~K}$

Fig. 7 a Configuration of the high speed machining; b Geometry of the rigid tool (a)

length $=0.002 \mathrm{~m}=2000 \mu \mathrm{m}$ high $=0.0002 \mathrm{~m}=200 \mu \mathrm{m}$ out of plane thickness $=0.000005 \mathrm{~m}=5 \mu \mathrm{m}$ rigid tool $\mathrm{v}=12 \mathrm{~m} / \mathrm{s}$

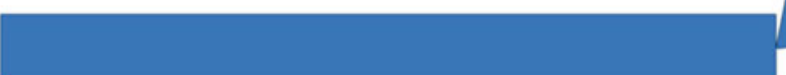

(b) angle $=7^{\circ}$

fillet radius $=3 \mu \mathrm{m}$

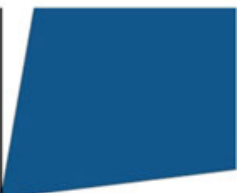

where $\theta$ is thermal expansion coefficient.

3. For viscoplastic flow phenomenon $\mathbf{d}^{p}[23,24]$

$\mathbf{d}^{p}=\Lambda^{v p} \mathbf{p}$,

where

$$
\begin{aligned}
& \Lambda^{v p}=\frac{1}{T_{m}}\left\langle\Phi^{v p}\left(\frac{f}{\kappa}-1\right)\right\rangle \\
& =\frac{1}{T_{m}}\left\langle\left(\frac{f}{\kappa}-1\right)^{m_{p l}}\right\rangle, \\
& f=\left\{J_{2}^{\prime}+\left[n_{1}(\vartheta)+n_{2}(\vartheta)(\xi: \xi)^{\frac{1}{2}}\right] J_{1}^{2}\right\}^{\frac{1}{2}}, \\
& n_{1}(\vartheta)=0, \quad n_{2}(\vartheta)=n=\text { const. }
\end{aligned}
$$

$$
\begin{aligned}
& \kappa=\left\{\kappa_{S}(\vartheta)-\left[\kappa_{S}(\vartheta)-\kappa_{0}(\vartheta)\right] \exp \left[-\delta(\vartheta) \in^{p}\right]\right\} \\
& {\left[1-\left(\frac{(\xi: \xi)^{\frac{1}{2}}}{\xi_{F}}\right)^{\beta(\vartheta)}\right] \text {, }} \\
& \bar{\vartheta}=\frac{\vartheta-\vartheta_{0}}{\vartheta_{0}}, \quad \kappa_{s}(\vartheta)=\kappa_{s}^{*}-\kappa_{s}^{* *} \bar{\vartheta}, \\
& \kappa_{0}(\vartheta)=\kappa_{0}^{*}-\kappa_{0}^{* *} \bar{\vartheta}, \quad \delta(\vartheta)=\delta^{*}-\delta^{* *} \bar{\vartheta}, \\
& \beta(\vartheta)=\beta^{*}-\beta^{* *} \bar{\vartheta}, \\
& \xi^{F}=\xi^{F^{*}}-\xi^{F^{* *}}\left\langle\left(\frac{\|\mathrm{L} \boldsymbol{v} \xi\|-\left\|\mathrm{L} \boldsymbol{v} \boldsymbol{\xi}_{c}\right\|}{\left\|\mathrm{L} \boldsymbol{v} \boldsymbol{\xi}_{c}\right\|}\right)^{m_{F}}\right\rangle, \\
& \mathbf{p}=\left.\frac{\partial f}{\partial \boldsymbol{\tau}}\right|_{\boldsymbol{\xi}=\text { const }}\left(\left\|\frac{\partial f}{\partial \boldsymbol{\tau}}\right\|\right)^{-1} \\
& =\frac{1}{\left[2 J_{2}^{\prime}+3 A^{2}(\operatorname{tr} \boldsymbol{\tau})^{2}\right]^{\frac{1}{2}}}\left[\boldsymbol{\tau}^{\prime}+A \operatorname{tr} \boldsymbol{\tau} \delta\right] \text {, }
\end{aligned}
$$




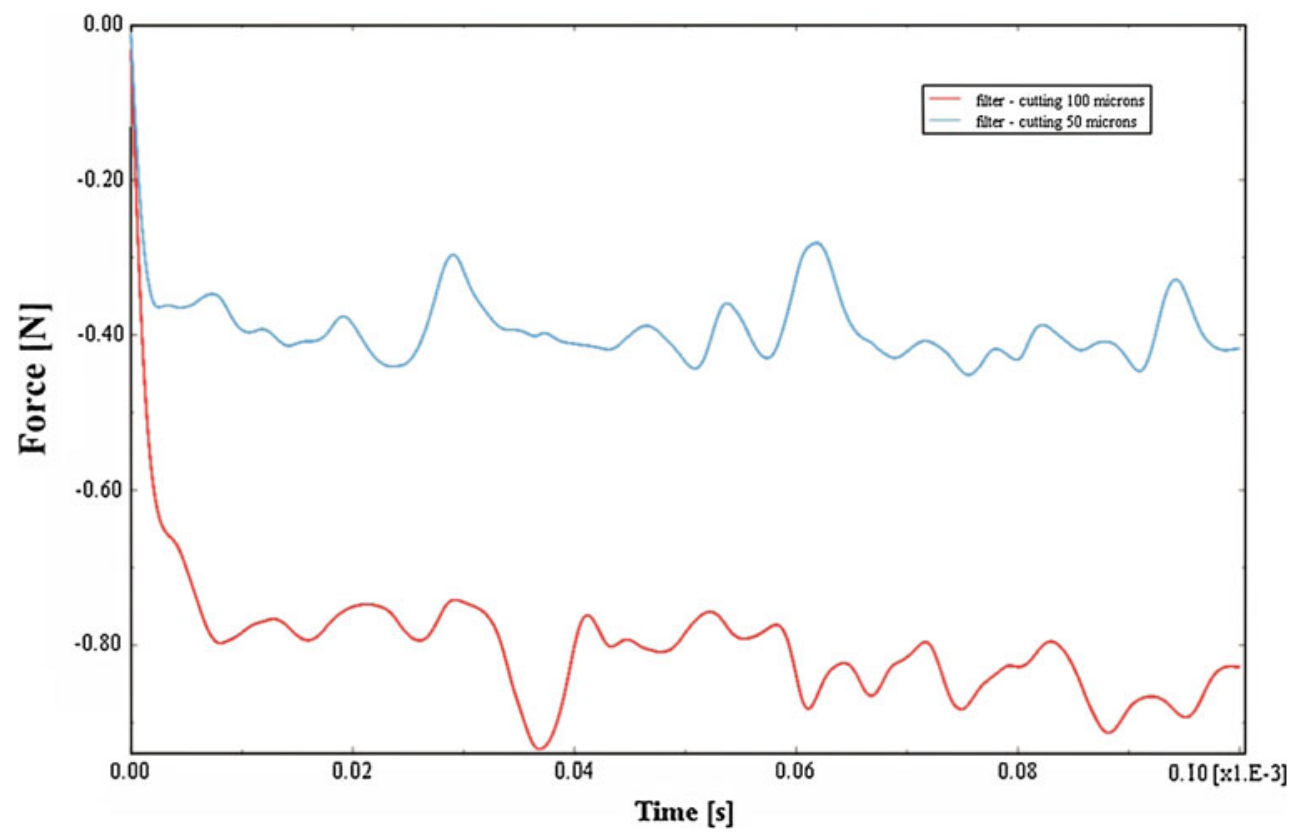

Fig. 8 The comparison of reaction on tool for cutting depths 50 and $100 \mu \mathrm{m}$

and $f$ denotes the potential function $[7,25,26,33], \kappa$ is the isotropic work hardening-softening function [17, 26], $\boldsymbol{\tau}^{\prime}$ represents the stress deviator, $J_{1}, J_{2}^{\prime}$ are the first and the second invariants of Kirchhoff stress tensor and deviatoric part of the Kirchhoff stress tensor, respectively, $A=2\left(n_{1}+n_{2}(\xi: \xi)^{\frac{1}{2}}\right), \xi^{F^{*}}$ can be thought as a quasi-static fracture porosity and $\left\|\mathrm{L} v \boldsymbol{\xi}_{c}\right\|$ denotes equivalent critical velocity of microdamage. Notice, that Eq. (19) reflects experimental fact, that the fracture porosity changes for fast processes. Such approach is consistent with the, so called, cumulative fracture criterion $[2,9,11]$, which assumes the existence of critical time needed for saturation of microdamage to its fracture limit.

4. For microdamage mechanism we take the additional assumptions $[3,7]$ :

- Velocity of the microdamage growth is coaxial with the principal directions of stress state,

- Only positive (tension) principal stresses induces the growth of the microdamage,

so we have

$$
\begin{aligned}
& \frac{\partial g^{*}}{\partial \boldsymbol{\tau}}=\left\langle\frac{\partial \hat{g}}{\partial \boldsymbol{\tau}}\right\rangle\left\|\left\langle\frac{\partial \hat{g}}{\partial \boldsymbol{\tau}}\right\rangle\right\|^{-1}, \quad \text { and } \hat{g}=\frac{1}{2} \boldsymbol{\tau}: \mathcal{G}: \boldsymbol{\tau} \\
& \Phi^{g}\left(\frac{I_{g}}{\tau_{e q}\left(\boldsymbol{\xi}, \vartheta, \in^{p}\right)}-1\right)=\left(\frac{I_{g}}{\tau_{e q}}-1\right)^{m_{g}}
\end{aligned}
$$

where

$$
\begin{aligned}
\tau_{e q}= & c(\vartheta)\left(1-(\xi: \xi)^{\frac{1}{2}}\right) \ln \frac{1}{(\xi: \xi)^{\frac{1}{2}}}\left\{2 \kappa_{s}(\vartheta)\right. \\
& \left.-\left[\kappa_{s}(\vartheta)-\kappa_{0}(\vartheta)\right] F(\xi 0, \xi, \vartheta)\right\}, \\
c(\vartheta)= & \text { const., } \\
F= & \left(\frac{\xi_{0}}{1-\xi_{0}} \frac{1-(\xi: \xi)^{\frac{1}{2}}}{(\xi: \xi)^{\frac{1}{2}}}\right)^{\frac{2}{3} \delta} \\
& +\left(\frac{1-(\xi: \xi)^{\frac{1}{2}}}{1-\xi_{0}}\right)^{\frac{2}{3} \delta}
\end{aligned}
$$

and

$I_{g}=\bar{b}_{1} J_{1}+\bar{b}_{2}\left(J_{2}^{\prime}\right)^{\frac{1}{2}}+\bar{b}_{3}\left(J_{3}^{\prime}\right)^{\frac{1}{3}}$.

$\bar{b}_{i}(i=1,2,3)$ are the material parameters, $J_{3}^{\prime}$ is the third invariant of deviatoric part of the Kirchhoff stress tensor.

Now, taking into account the postulates for microdamage evolution, and assuming that tensor $\mathcal{G}$ can be written as a symmetric part of the fourth order unity tensor $\mathcal{I}$ [20]

$\mathcal{G}=\mathcal{I}^{s}, \quad \mathcal{G}_{i j k l}=\frac{1}{2}\left(\delta_{i k} \delta_{j l}+\delta_{i l} \delta_{j k}\right)$,

we can write the explicit form of the growth function $\hat{g}$ as

$$
\hat{g}=\frac{1}{2}\left(\tau_{I}^{2}+\tau_{I I}^{2}+\tau_{I I I}^{2}\right) .
$$


cutting depth $=50 \mu \mathrm{m}$

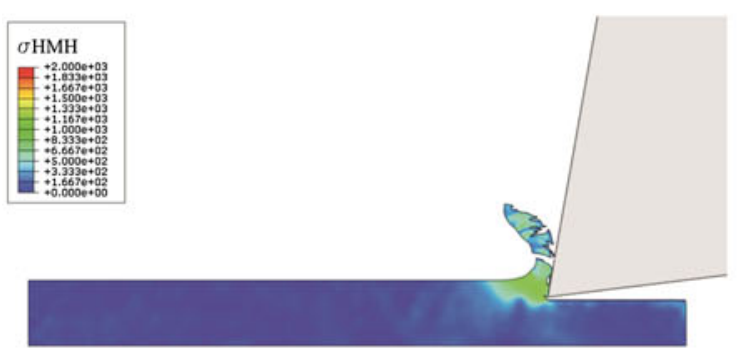

Step Time $=3.5000 \mathrm{E}-05$
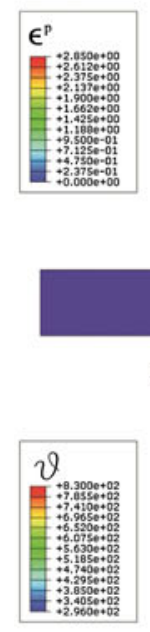

Step Time $=3.5000 \mathrm{E}-05$

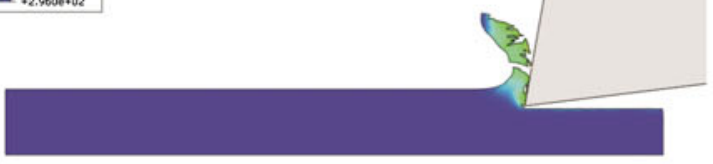

Step Time $=3.5000 \mathrm{E}-05$
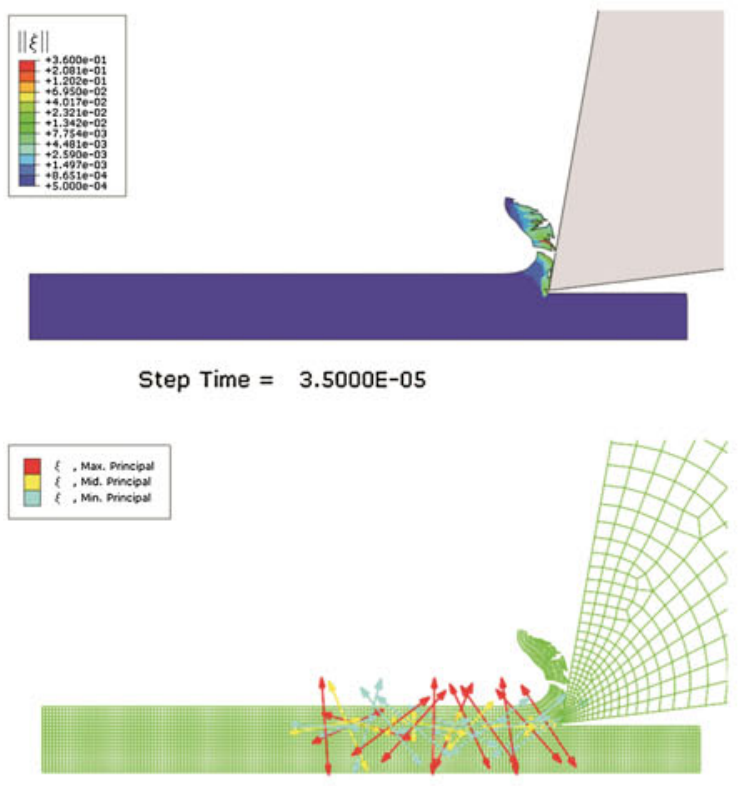

Step Time $=3.5000 \mathrm{E}-05$ cutting depth $=100 \mu \mathrm{m}$

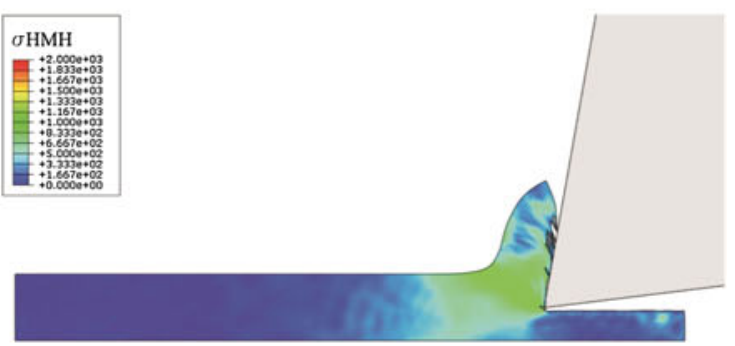

Step Time $=3.5000 \mathrm{E}-05$

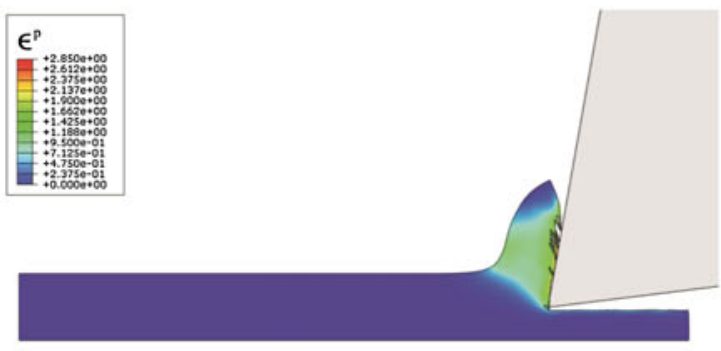

$:$ Step Time $=3.5000 \mathrm{E}-05$

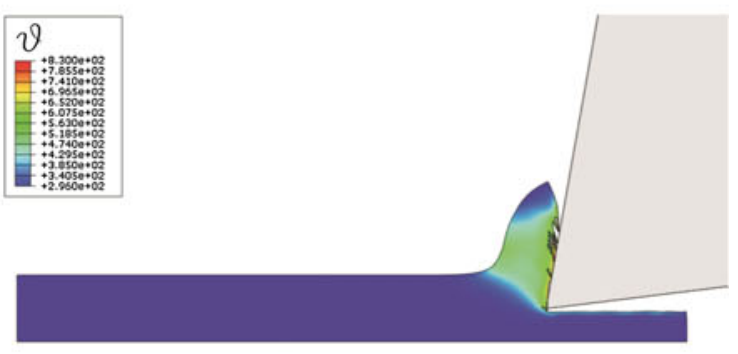

Step Time $=3.5000 \mathrm{E}-05$
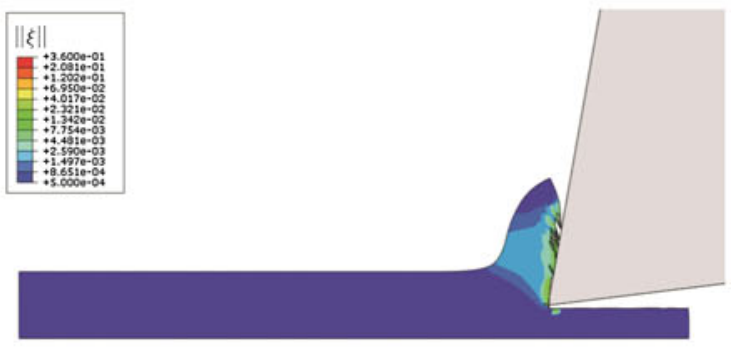

Step Time $=3.5000 \mathrm{E}-05$
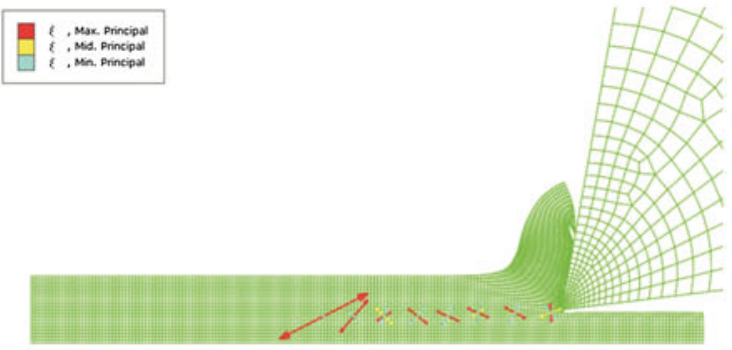

Step Time $=3.5000 \mathrm{E}-05$

Fig. 9 The comparison of the numerical results for cutting depths $50 \mu \mathrm{m}$ (left column) and $100 \mu \mathrm{m}$ (right column) at time instant $3.5 \cdot 10^{-5} \mathrm{~s}$ for cutting speed $12 \mathrm{~ms}^{-1}$ 
cutting depth $=50 \mu \mathrm{m}$

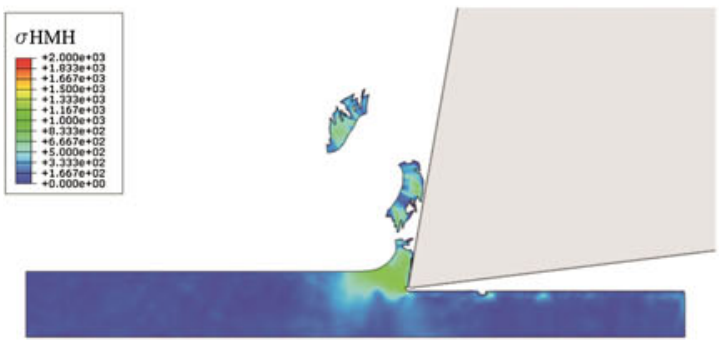

Step Time $=7.0000 \mathrm{E}-05$
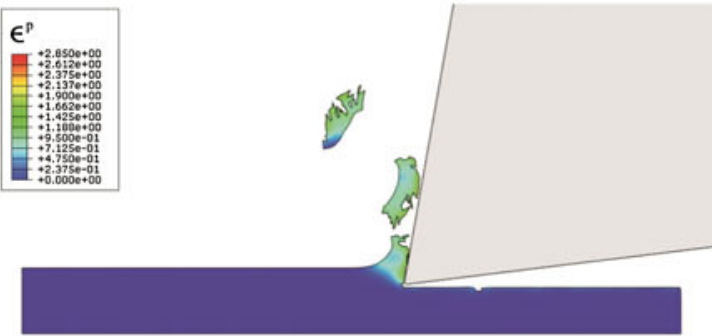

Step Time $=7.0000 \mathrm{E}-05$
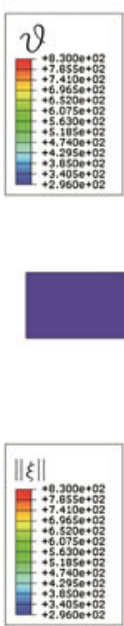

Step Time $=7.0000 \mathrm{E}-05$

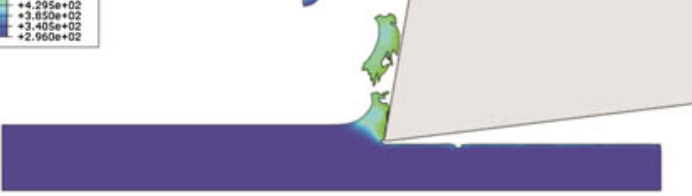

Step Time $=7.0000 \mathrm{E}-05$
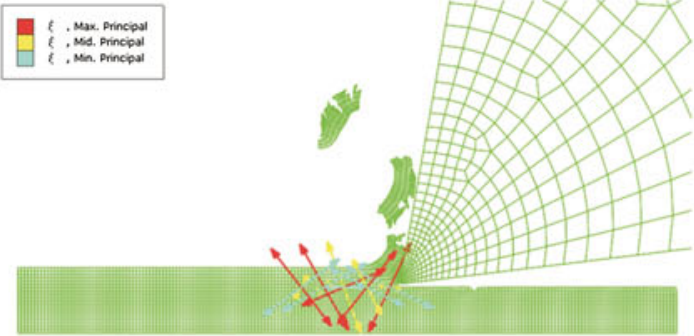

Step Time $=7.0000 \mathrm{E}-05$ cutting depth $=100 \mu \mathrm{m}$
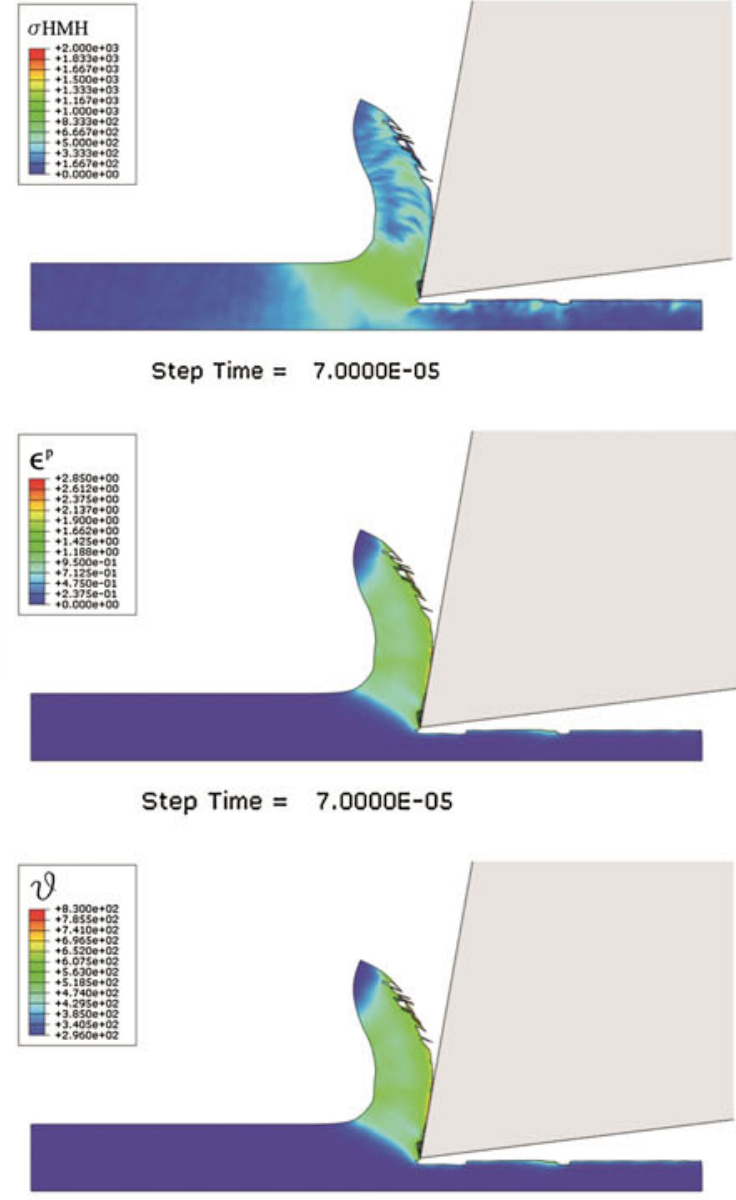

Step Time $=7.0000 \mathrm{E}-05$

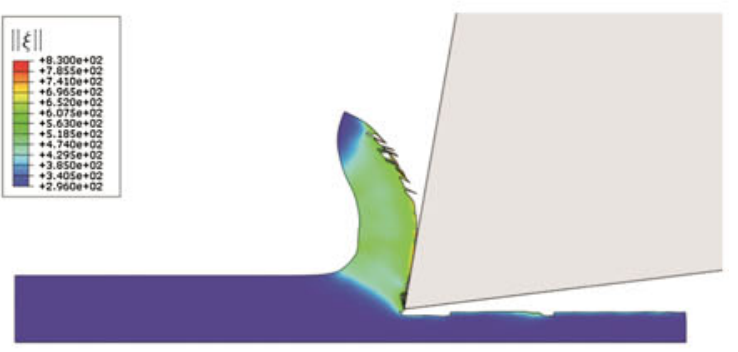

Step Time $=7.0000 \mathrm{E}-05$
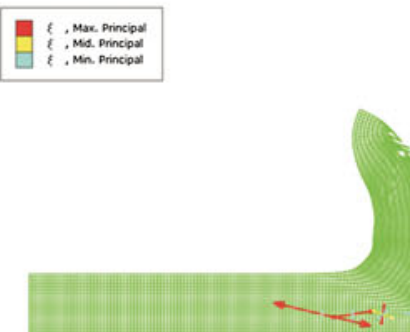

Step Time $=7.0000 \mathrm{E}-05$

Fig. 10 The comparison of the numerical results for cutting depths $50 \mu \mathrm{m}$ (left column) and $100 \mu \mathrm{m}$ (right column) at time instant $7.0 \cdot 10^{-5} \mathrm{~s}$ for cutting speed $12 \mathrm{~ms}^{-1}$ 
cutting depth $=50 \mu \mathrm{m}$

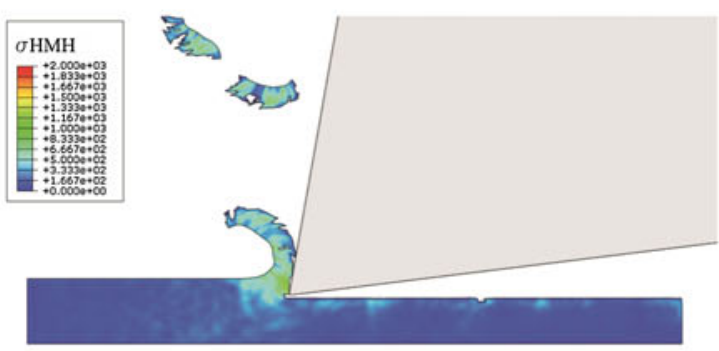

Step Time $=1.0000 \mathrm{E}-04$
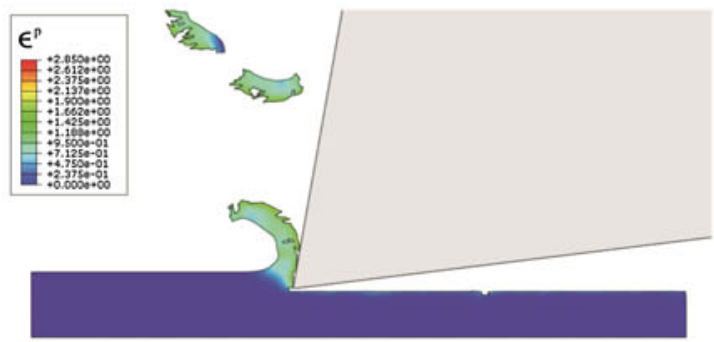

Step Time $=1.0000 \mathrm{E}-04$
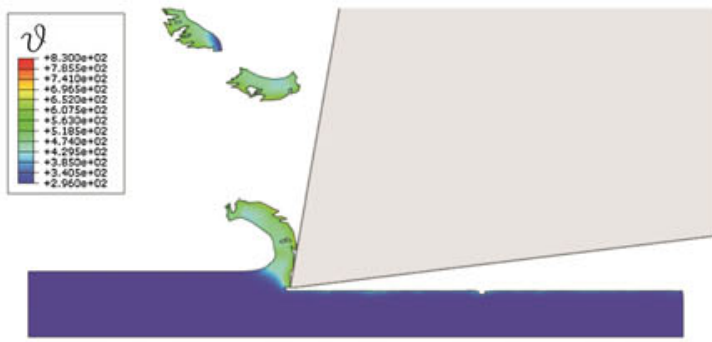

Step Time $=1.0000 \mathrm{E}-04$
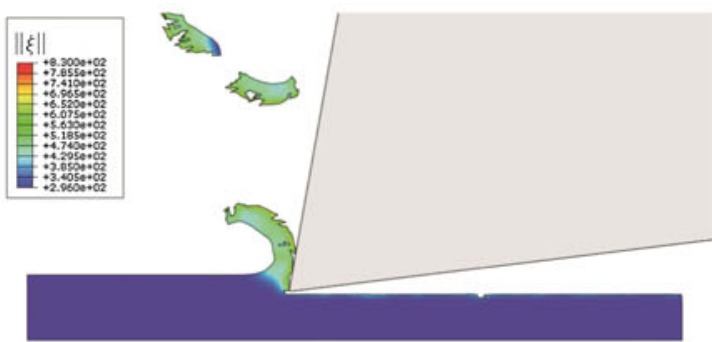

Step Time $=1.0000 \mathrm{E}-04$

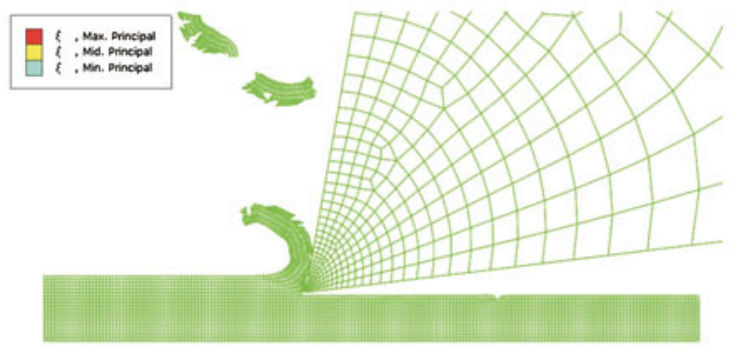

Step Time $=1.0000 \mathrm{E}-04$ cutting depth $=100 \mu \mathrm{m}$

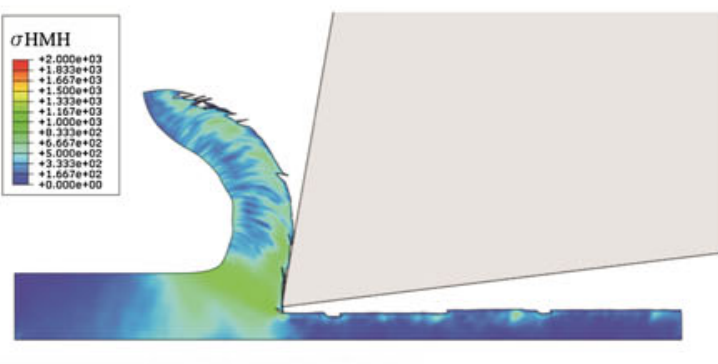

Step Time $=1.0000 \mathrm{E}-04$

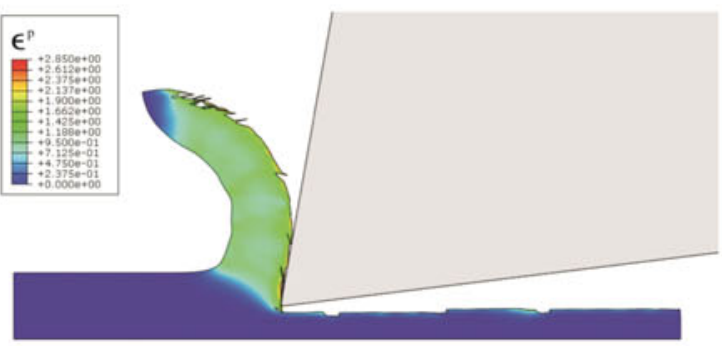

Step Time $=1.0000 \mathrm{E}-04$

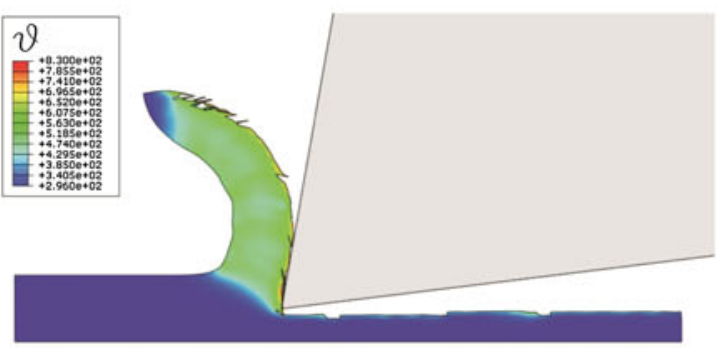

Step Time $=1.0000 \mathrm{E}-04$

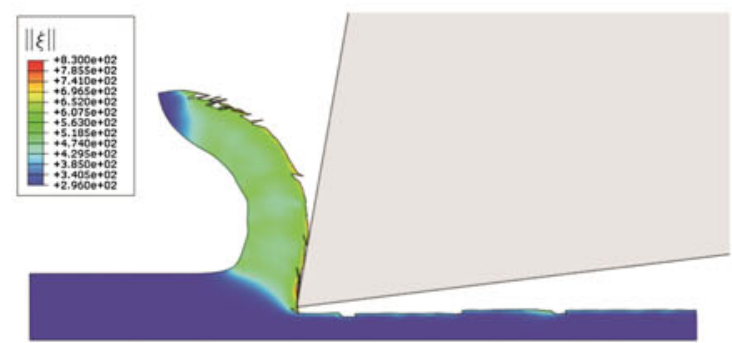

Step Time $=1.0000 \mathrm{E}-04$

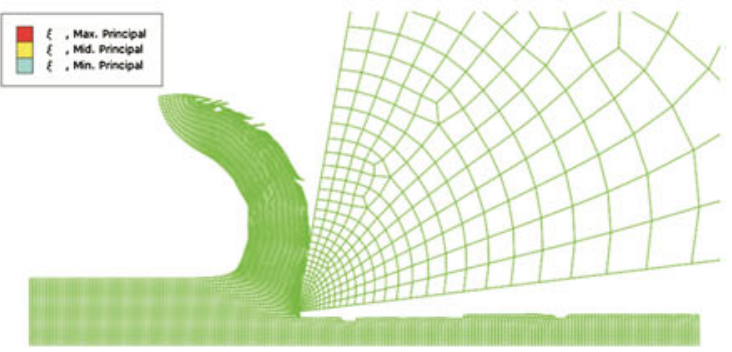

Step Time $=1.0000 \mathrm{E}-04$

Fig. 11 The comparison of the numerical results for cutting depths $50 \mu \mathrm{m}$ (left column) and $100 \mu \mathrm{m}$ (right column) at time instant $1.0 \cdot 10^{-4} \mathrm{~s}$ for cutting speed $12 \mathrm{~ms}^{-1}$ 
The gradient of $\hat{g}$ with respect to the stress field gives us the following matrix representation of a tensor describing the anisotropic evolution of microdamage

$$
\frac{\partial \hat{g}}{\partial \boldsymbol{\tau}}=\left[\begin{array}{ccc}
g_{11} \tau_{I} & 0 & 0 \\
0 & g_{22} \tau_{I I} & 0 \\
0 & 0 & g_{33} \tau_{I I I}
\end{array}\right] .
$$

In (28) $\tau_{I}, \tau_{I I}, \tau_{I I I}$ are the principal values of Kirchhoff stress tensor.

It can be noted, that the definition of the threshold stress for microcrack growth function $\tau_{e q}$ indicates that the growth term in evolution function for microdamage is active only after nucleation whereas before nucleation we have infinite threshold $\lim _{\boldsymbol{\xi} \rightarrow \mathbf{0}} \tau_{e q}=\infty$.

5. For temperature evolution Eq. (10) we consider the following relation

$$
\mathbf{k}=\boldsymbol{\tau} .
$$

\section{ANN approximation}

\subsection{Number of material parameters - summary}

The adiabatic process, as outlined above, in terms of the discussed model requires identification of 28 material parameters [35]. We propose to split them as follows:

- Five parameters related to viscoplastic evolution (including five dimensionless) $-m_{p l}, \beta^{*}, \beta^{* *}, n_{1}, n_{2}$,

- Nine parameters related to anisotropic microdamage evolution (including eight dimensionless) $-m_{g}, c, b_{1}, b_{2}$, $b_{3}, \xi^{F^{*}}, \xi^{F^{* *}}, m_{F},\left\|\mathrm{~L}_{\boldsymbol{v}} \boldsymbol{\xi}_{c}\right\|$

- Seven parameters shared with viscoplastic and microdamage evolution (including two dimensionless) $-T_{m}$, $\kappa_{s}^{*}, \kappa_{s}^{* *}, \kappa_{0}^{*}, \kappa_{0}^{* *}, \delta^{*}, \delta^{* *}$,

- Four parameters related to thermal evolution (including two dimensionless $)-\chi^{*}, \chi^{* *}, \theta, c_{p}$,

- Two elasticity parameters- $-\lambda, \mu$, and

- reference density- $\rho_{\text {Ref }}$,

with all parameters being non negative. In [35] it was shown that without loss of model's generality one can reduce this number to 22 . Next, we present further reduction in number of parameters based on ANN approximation. The reduction consists of work hardening-softening and threshold stress for microcrack growth functions which in our opinion do not cause the loss of generality of formulation nor any mathematical consequences. It gives, as mentioned, final amount of a material parameters equal 19 to handle particular metal behaviour.

\subsection{Approximation concept}

The concept of ANN approximation of work hardening-softening and threshold stress for microcrack growth functions can be summarised as follows.

One can rewrite the work hardening-softening function Eq. (17) as

$$
\begin{aligned}
\kappa= & \kappa\left(\in^{p}, \vartheta, \boldsymbol{\xi}\right)=\left\{1-\left[\frac{(\boldsymbol{\xi}: \boldsymbol{\xi})^{\frac{1}{2}}}{\xi_{F}}\right]^{\beta(\vartheta)}\right\}\left\{\kappa_{S}(\vartheta)\right. \\
& \left.-\left[\kappa_{s}(\vartheta)-\kappa_{0}(\vartheta)\right] \exp \left[-\delta(\vartheta) \in^{p}\right]\right\},
\end{aligned}
$$

and threshold stress for microcrack growth function Eq. (23) as

$$
\begin{aligned}
\tau_{e q}= & \tau_{e q}\left(\in^{p}, \vartheta, \boldsymbol{\xi}\right)=2 c(\vartheta)\left(1-(\xi: \xi)^{\frac{1}{2}}\right) \ln \frac{1}{(\xi: \xi)^{\frac{1}{2}}} \\
& \left\{\kappa_{s}(\vartheta)-\frac{1}{2}\left[\kappa_{s}(\vartheta)-\kappa_{0}(\vartheta)\right] F\left(\xi_{0}, \xi, \vartheta\right)\right\} .
\end{aligned}
$$

Now we propose the following approximations for both functions

$\kappa^{*}=\kappa\left(\in^{p}, \dot{\epsilon}^{p}, \vartheta, \boldsymbol{\xi}\right)=\xi_{\kappa} \mathcal{A}, \quad$ where

$\xi_{\kappa}=1-\left[\frac{(\xi: \xi)^{\frac{1}{2}}}{\xi_{F}}\right]^{\beta(\vartheta)}$,

and

$\tau_{e q}^{*}=\tau_{e q}\left(\epsilon^{p}, \dot{\epsilon}^{p}, \vartheta, \xi\right)=\xi_{e q} \mathcal{A}, \quad$ where

$\xi_{e q}=\left(1-(\xi: \xi)^{\frac{1}{2}}\right) \ln \frac{1}{(\xi: \xi)^{\frac{1}{2}}}$,

where $\mathcal{A}$ is an ANN approximation which keeps the effects of equivalent plastic strain, strain rates and temperature on flow stress (from experiment), while functions $\xi_{\kappa}, \xi_{e q}$ introduce the phenomenological scaling only due to anisotropic microdamage effect. Hence, after ANN learning process, $\mathcal{A}$ it is a function which for known values of $\in^{p}, \dot{\epsilon}^{p}, \vartheta$ gives the current value of flow stress in a material point which needs to be scaled by $\xi_{\kappa}, \xi_{e q}$ to introduce microdamage effects. It is important to notice, that the structure of Eqs (32) and (33) is in agreement with previously used explicit functions [35] which were obtained from micromechanics considerations at the level of a single void [3].

By eliminating $\kappa_{s}, \kappa_{0}$ and $\delta$ from material functions in fact we reduce the number of material parameters by $6 \mathrm{cf}$. Eqs $(18)_{2},(18)_{3},(18)_{4}$.

The comment requires the introduction of strain rate $\left(\dot{\epsilon}^{p}\right)$ in both approximated functions. Originally in the Perzyna's formulation functions $\kappa$ and $\tau_{e q}$ are quasi-static while the strain rate effects come from a relaxation time $\left(T_{m}\right)$ which is a function of $\dot{\epsilon}^{p}$. Such a concept has a deep physical interpretation derived from the analysis of a single crystal and a polycrislal behavior (cf. [29]). Nevertheless, in the concept 
presented in this paper it is convenient to introduce a strain rate in $\kappa^{*}$ and $\tau_{e q}^{*}$.

Finally, the assumption in the presented formulation of no initial porosity $\xi_{0}=0$ leads to $\tau_{e q}^{*} \rightarrow \infty$. For such case, according to Sect. 3.1, we reduce our material parameters by 9 and only 10 parameters for a specific steel remain. In most of the times they can be relatively easy taken from the literature - apart from the data needed for ANN learning (strainstress curves for versus temperatures and strain rates). Such a case is not considered further in the paper.

The details concerning implementation can be found in e.g. $[35,37]$-moreover it must be emphasized that the implementation keeps the Lie objective rate.

\section{Numerical examples}

\subsection{Material model implementation}

The discussed material model is implemented in Abaqus/ Explicit finite element code by taking advantage of a user subroutine VUMAT, which is coupled with Abaqus system [1]. It's important to mention that Abaqus/Explicit utilizes central-difference time integration rule along with the diagonal ("lumped") element mass matrices. We use so called element deletion method to remove damaged elements from the mesh [34] —elements in which for every integration point fracture porosity was reached.

\subsection{Identification of DH-36 steel parameters}

The experimental results are taken from [15]. In [15] the authors investigated the behavior of DH-36 steel for three different strain rates $\left(0.001,0.1\right.$ and $\left.3,000 s^{-1}\right)$ and for the sake of comparison for six different temperatures $(77,296,400,500,600$ and $800 K)$. The DH-36 steel belongs to the class of high strength structural low-alloy steels. The DH-36 steel distinguishes itself due to good weldability, toughness, good ductility and plasticity (true strain $>60 \%$ ) under various temperatures and loading rates. The steel has the characteristics of the bcc structure, hence it belongs to the so called ferritic steels. The mechanical properties of the DH-36 steel are strongly affected by impurities in its internal structure. It is important, that the processing (rolling) of the DH-36 steel, can induce the anisotropy of its structure [8].

In order to finalize the overall concept we must assume that such experimental data set is sufficient to cover all characteristic phenomena for phenomenological description of analyzed steel. However, it should be emphasized, that in our case for modelling of extremely fast thermomechanical processes, the range of strain rates and simultaneously temperatures being analyzed should be increased. It is due to the fact that it would guarantee that not only the approximation but also extrapolation from ANN will be conducted with acceptable errors.

We used the ANN with single hidden layer (16 neurons) using constrained truncated Newton algorithm for learning. The input consisted of equivalent plastic strain, strain rate and temperature while the target consisted of corresponding flow stress. Both input and output were directly taken from the experimental (isothermal) curves, as provided in [15]. The results of approximation/extrapolation are presented in Figs 2, 3, 4 and 5.

In Fig. 2 we present the original data set (isothermal) taken from [15] and its ANN approximation-please notice, that the approximation is performed with negligible error. Finally, since the analysis of a 3D body under extreme loading conditions leads to a broad range of strains and temperature localizations we need to provide a good extrapolation both in strains and temperature axes as shown in Figs 3 and 4.

Figure 5 representing the extrapolation through the strain rates, requires separate discussion. Due to the fact that the experimental results cover only $0.001,0.1$ and $3,000 \mathrm{~s}^{-1}$ rates, the ANN extrapolation can lead to nonphysical answer (cf. plot for 10, $000 \mathrm{~s}^{-1}$ in Fig. 5). Therefore, during computations we assumed three strain ranges $0 \div 0.001 \mathrm{~s}^{-1}$, $0.001 \div 1 \mathrm{~s}^{-1}$ and $>1 \mathrm{~s}^{-1}$. In case during the analysis the strain rate was in one of those ranges we induce to take the values from the flow surface for rates $0.001,0.1$ and $3,000 \mathrm{~s}^{-1}$, respectively.

Finally, in Table 1 we present a complete set of parameters, identified in the sense of numerical calibration, for analyzed steel. Note, that material parameters that are reduced are marked within a box in Table 1 . Intentionally we additionally omitted three more parameters $m_{F},\left\|\mathrm{~L} v \xi_{c}\right\|, \xi^{F^{* *}}$ assuming that fracture porosity in constant.

In Fig. 6 the comparison of the strain-stress curves (isothermal and adiabatic) from the experiment and the model for a strain rate $3,000 \mathrm{~s}^{-1}$ and the initial temperature $296 \mathrm{~K}$ are provided as an example. The adiabatic curve given by the model is obtained from 3D simulation being a model mapping experimental set up [15] while the isothermal one is from ANN.

\subsection{Orthogonal cutting analysis}

\subsubsection{Geometry}

The analyzed HSM set up is presented in Fig. 7. The tool is rigid and its geometry is described be an exterior angle equals to $7^{\circ}$ and the fillet radius equals to $3 \mu \mathrm{m}$. The dimensions of the workpiece (3D) are length 2, $000 \mu \mathrm{m}$, height $200 \mu \mathrm{m}$ and out of plane thickness $5 \mu \mathrm{m}$.

The boundary conditions on a workpiece are applied at the bottom, front and rear surfaces, while rigid tool can move in 
horizontal direction only with constant velocity $12 \mathrm{~ms}^{-1}$. The initial conditions assumed room temperature.

We used 8-node linear brick, reduced integration element (C3D8R) with approximate size $10 \mu \mathrm{m}$.

Friction coefficient between surfaces in contact is assumed to be equal 0.3 .

\subsubsection{Results}

\section{Global analysis}

The comparison of the reaction on a tool for cutting depth 50 and $100 \mu \mathrm{m}$ is presented in Fig. 8. It is important to recall, that those results are the most important for machinery designers. We observe that an average force that acts on the tool for cutting depth $50 \mu \mathrm{m}$ is around $0.4 \mathrm{~N}$ while for cutting depth $100 \mu \mathrm{m}$ it reaches approximately $0.8 \mathrm{~N}$.

\section{Local analysis}

The complete local analysis of orthogonal cutting problem comprises of: creation of shear bands, machined surface roughness, level of plastic strains, local temperature, chip thickness etc. Nevertheless, we regard this numerical example as a benchmark since our analysis are simplified.

The comparison of the HMH stresses, the equivalent viscoplatic strains, the temperature, the porosity maps and privileged damage directions for cutting depths 50 and $100 \mu \mathrm{m}$ at time instant $3.5 \cdot 10^{-5}, 7.0 \cdot 10^{-5}, 10^{-4} \mathrm{~s}$ are presented in Figs 9, 10 and 11, respectively. Note, that for easier analysis of the maps, each time in rows we use legend with the same upper and lower bounds.

The first look shows severe fragmentation of a chip for cutting depths $50 \mu \mathrm{m}$. Likewise in the experiment (cf. [14]) peak stresses are near tool tip and their spatial distribution properly maps the ability for shear band formation. The equivalent plastic strains can be even 2, while local temperature increase is close to $450 \mathrm{~K}$. The chip is thicker than initial cut depth.

Let us now discuss the results coming from anisotropy of damage which is described be the model. Recall, that we use second order tensor to describe directional behavior of damage (microdamage tensor $\boldsymbol{\xi}$ ) and also the physical interpretation of $\boldsymbol{\xi}$ is that the Euclidean norm of the microdamage field defines the scalar quantity called porosity while its principal values indicate damage plane as one perpendicular to maximal principal value of $\boldsymbol{\xi}$.

In Figs 9, 10 and 11 porosity maps and plots of principal directions of $\boldsymbol{\xi}$ (in selected material points) are presented. Initial value of porosity for metals circulates around $6 \cdot 10^{-4}[3,16,18]$ while fracture porosity in our case is equal to 0.3 . That is why it is hard to present such quantity on contour maps, hence we use logarithmic scale (see legend for porosity). However porosity does not provide any informa- tion regarding the damage directions. The situation is different when we analyse principal directions of $\boldsymbol{\xi}$, remembering that damage plane is one perpendicular to maximal principal value (red arrow on plot). Due to elasto-viscoplastic waves traveling during simulation through the workpiece those are the possible directions of creation new surface changes. However, it is clearly visible that close to the tip of the tool they always show correct direction of macrodamage propagation. Hence using the presented material model, we can trace the direction of softening, and predict damage pattern.

\section{Conclusions}

In this paper the reduction of material parameters by using ANN approximation for selected material functions plays the key part. The material model chosen for application of the ANN approximation concept is described by recently proposed Perzyna's type viscoplasticity including anisotropic measure of damage $[5,29,35]$. This model is designed for metals under extreme loading conditions and originally introduces 28 material parameters for analysis under adiabatic conditions. In the paper we show that this number of material parameters can be reduced to 19 using the concept of ANN approximation. It is important that the introduced approximation does not violate proper understanding of fundamental phenomena derived from analysis of a single crystal and a polycrislal behavior postulates originally by Perzyna (cf. [28]).

Apart the concept of ANN approximation itself, we are providing the short description of the material model which is used as an example, its implementation in Abaqus/Explicit finite element software and application to HSM problems. Finally we can conclude that ANN approximation is very effective, however considering numerical analysis of high speed phenomena which covers a broad range of temperature (up to melting point), strain rates (up to $10^{7} \mathrm{~s}^{-1}$ ), strains (up to 2) etc. the experimental collection of data must be large. It is due to the fact that the answer from ANN (in particular extrapolated values) depends on data set used for learning.

Acknowledgments The support of the Polish Ministry of Science and Higher Education under Grant R00 009712 is kindly acknowledged.

Open Access This article is distributed under the terms of the Creative Commons Attribution License which permits any use, distribution, and reproduction in any medium, provided the original author(s) and the source are credited.

\section{References}

1. Abaqus Version 6.12 Theory manual. 2012

2. Campbell JD (1953) The dynamic yielding of mild steel. Acta Metall 1(6):706-710 
3. Dornowski W (1999) Influence of finite deformations on the growth mechanism of microvoids contained in structural metals. Arch Mech 51(1):71-86

4. Eftis J, Carrasco C, Osegueda RA (2003) A constitutivemicrodamage model to simulate hypervelocity projectile-target impact, material damage and fracture. Int J Plasticity 19:13211354

5. Glema A, Łodygowski T, Sumelka W (2010) Nowacki's double shear test in the framework of the anisotropic thermo-elastovicsoplastic material model. J Theor Appl Mech 48(4):973-1001

6. Glema A, Łodygowski T, Sumelka W (2010) Towards the modelling of an anisotropic solids. Comput Methods Sci Technol 16(1):73-84

7. Glema A, Łodygowski T, Sumelka W, Perzyna P (2009) The numerical analysis of the intrinsic anisotropic microdamage evolution in elasto-viscoplastic solids. Int J Damage Mech 18(3): 205-231

8. Hu LH, Huang J, Li ZG, Wu YX (2011) Effects of preheating temperature on cold cracks, microstructures and properties of high power laser hybrid welded $10 \mathrm{Ni} 3 \mathrm{CrMoV}$ steel. Mater Design 32(4):1931-1939

9. Jankowiak T, Klepaczko JR, Łodygowski T (2006) Numerical modeling of wave propagation and interaction in bars. Foundations of Civil and Environmental Engineering 7(4):187-199

10. Johnson GR, Cook WH (1983) A constitutive model and data for metals subjected to large strains, high strain rates and high temperatures. In: Proceedings of the seventh international symposium on ballistics, Hague, The Netherlands, p 541-547

11. Klepaczko JR (1990) Behavior of rock like materials at high strain rates in compression. Int J Plasticity 6:415-432

12. Lee EH (1969) Elastic-plastic deformation at finite strain. ASME J Appl Mech 36:1-6

13. Marsden JE, Hughes TJH (1983) Mathematical foundations of elasticity. Prentice-Hall, New Jersey

14. Molinari A, Musquar C, Sutter G (2002) Adiabatic shear banding in high speed machining of $\mathrm{Ti}-6 \mathrm{Al}-4 \mathrm{~V}$ : experiments and modeling. Int J Plasticity 18:443-459

15. Nemat-Nasser S, Guo W-G (2003) Thermomechanical response of DH-36 steel plates over a wide range of strain rates and temperatures. Mech Mater 35:1023-1047

16. Nemes JA, Eftis J (1991) Several features of a viscoplastic study of plate-impact spallation with multidimensional strain. Comput Struct 38(3):317-328

17. Nemes JA, Eftis J (1993) Constitutive modelling of the dynamic fracture of smooth tensile bars. Int J Plasticity 9(2):243-270

18. Nemes JA, Eftis J, Randles PW (1990) Viscoplatic constitutive modelling of high strain-rate deformation, material damage and spall fracture. J Appl Mech 57:282-291

19. Łodygowski $T$ (1996) Theoretical and numerical aspects of plastic strain localization. D.Sc. Thesis, volume 312, Poznan University of Technology

20. Łodygowski T, Glema A, Sumelka W (2008) Anisotropy induced by evolution of microstructure in ductile material. In: 8th World congress on computational mechanics (WCCM8), 5th European congress on computational methods in applied sciences and engineering (ECCOMAS 2008), Venice, 30 June-5 July 2008
21. Łodygowski T, Perzyna P (1997) Localized fracture of inelastic polycrystalline solids under dynamic loading process. Int J Damage Mech 6:364-407

22. Łodygowski T, Perzyna P (1997) Numerical modelling of localized fracture of inelastic solids in dynamic loading process. Int $\mathrm{J}$ Num Methods Eng 40:4137-4158

23. Perzyna $P$ (1963) The constitutive equations for rate sensitive plastic materials. Quart Appl Math 20:321-332

24. Perzyna P. (1966) Fundamental problems in viscoplasticity. Adv Appl Mech 9:243-377

25. Perzyna $P$ (1986) Constitutive modelling for brittle dynamic fracture in dissipative solids. Arch Mech 38:725-738

26. Perzyna $P(1986)$ Internal state variable description of dynamic fracture of ductile solids. Int J Solids Struct 22:797-818

27. Perzyna $P$ (1994) Instability phenomena and adiabatic shear band localization in thermoplastic flow process. Acta Mech 106: 173-205

28. Perzyna P (2005) The thermodynamical theory of elastoviscoplasticity. Eng Trans 53:235-316

29. Perzyna P (2008) The thermodynamical theory of elastoviscoplasticity accounting for microshear banding and induced anisotropy effects. Mechanics 27(1):25-42

30. Rusinek A, Klepaczko JR (2009) Experiments on heat generated during plastic deformation and stored energy for trip steels. Mater Design 30(1):35-48

31. Rusinek A, Rodríguez-Martínez JA (2009) Thermo-viscoplastic constitutive relation for aluminium alloys, modeling of negative strain rate sensitivity and viscous drag effects. Mater Design 30(10):4377-4390

32. Rusinek A, Rodriguez-Martinez JA, Klepaczko JR, Pęcherski RB (2009) Analysis of thermo-visco-plastic behaviour of six high strength steels. Mater Design 30(5):1748-1761

33. Shima S, Oyane M (1976) Plasticity for porous solids. Int J Mech Sci 18:285-291

34. Song J-H, Wang H, Belytschko T (2008) A comparative study on finite element methods for dynamic fracture. Comput Mech 42:239-250

35. Sumelka W (2009) The constitutive model of the anisotropy evolution for metals with microstructural defects. Publishing House of Poznan University of Technology, Poznan

36. Sumelka W, Glema A (2009) Theoretical and computational aspects of implementation of anisotropic constitute model for metals with microstructural defects. In: 18th International conference on computer methods in mechanics CMM-2009, p 451-452, Zielona Góra, Poland, 18-21 May 2009

37. Sumelka W, Łodygowski T (2011) The influence of the initial microdamage anisotropy on macrodamage mode during extremely fast thermomechanical processes. Arch Appl Mech 81(12): 1973-1992

38. Truesdell C, Noll W (1965) The non-linear field theories of mechanics. In: Flügge S (ed) Handbuch der Physik, vol 3. Springer, Berlin 